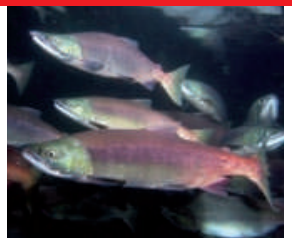

SHOCK SALMON GLUT

34 million fish throng

Canadian river.

go.nature.com/7vv9Pf

\title{
Teams battle for neuron prize
}

Contest spurs progress for programs that can map a brain cell's myriad branches.

Flanked by his graduate students, Badrinath Roysam, a computer scientist from the Rensselaer Polytechnic Institute in Troy, New York, stared tight-lipped at a large computer screen covered in strange white cobwebs and thin lines. A judge sat upright in a chair, pointing out gaps and mistakes in the data on the screen. A timekeeper tapped at an electronic stopwatch.

Down the hall at the Janelia Farm Research Campus in Ashburn, Virginia, part of the Howard Hughes Medical Institute, four other teams were also being judged under the watchful eyes of timekeepers. They were taking part in the final round of the Digital Reconstruction of Axonal and Dendritic Morphology (DIADEM) Challenge, which ran from 29 August to 1 September. The contest aimed to promote the development of computer algorithms that can successfully chart a neuron's many twists and branches; a time-consuming task that has plagued neurobiologists for decades. In the process, it has pioneered a novel way to advance biomedical innovation - through competitions and prizes.

Neuron shape is intimately related to function, and mapping it is key to tracing the myriad connections that make up a working nervous system. For biologists, tracing each forking axon and dendrite is straightforward but slow, requiring researchers to painstakingly work their way through stacks of microscope images. It can take six months to map the hundreds or thousands of branches in a single rat cortex neuron by eye - the entire cortex could take millions of years. Computer scientists, by contrast, say that their algorithms should make quick work of the problem - except that the programs can only cope with images that are simple enough for a computer to interpret.

"In creating this challenge, I imagined these two groups sparring around a chessboard," says the competition's organizer, Giorgio Ascoli, a neuroscientist at George Mason University in Fairfax, Virginia. He says that the contest's objective was to force the two sides to engage, pushing biologists to make their data more computer friendly, and making algorithms robust enough to contend with real-world information.

The DIADEM challenge asked teams of

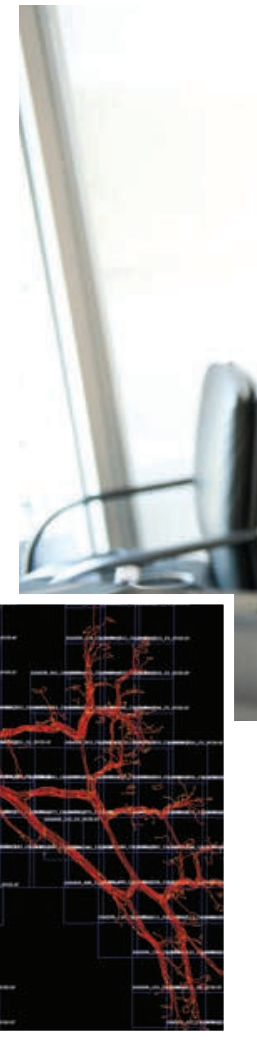
the sets of images thrown at the competing algorithms were intended to be suitably tough and were drawn from many different organisms, brain regions, cell types and staining techniques. The neurologists who provided the data also acted as judges. For added impetus, a US\$75,000 prize was offered.

Yet the challenge proved more difficult than expected. Five of the 125 teams that entered algorithms in the competition made it to last week's finals, but none achieved the benchmark of a 20-fold improvement in speed. In the end, the judges decided to divide the prize money between four of the teams - including Roysam's - based on which algorithms showed the most promise.

Despite having no clear winner, the tournament ended with plenty of positive momentum, says Ascoli. Minutes after the prizes were announced, contestants proposed the formation of a consortium to advance the technology. "I thought this would be in the future, but this event made it start to happen now," says Ascoli.

The DIADEM challenge also spurred innovation for a fraction of its normal cost. computer scientists to find accurately map a single neuron's branches at least 20 times faster than a human. Ascoli says that

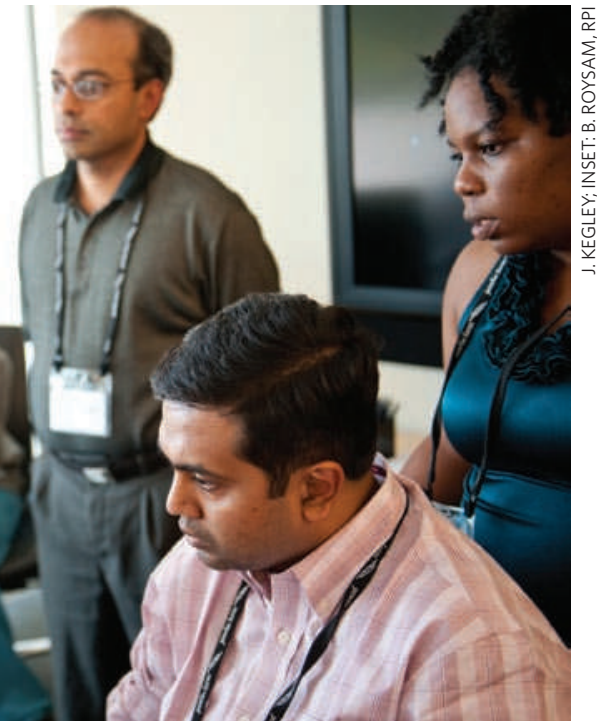

Badrinath Roysam (centre, standing) and his team developed an algorithm to (n) National Institutes of Health (NI).

The US Defense Advanced Research Projects Agency has often used prizes to encourage innovation. In upcoming legislation, the National Science Foundation (NSF) is also likely to be granted this authority from Congress - a necessary step because it represents a variation from the NSF mandate to use peer review to guide fund allocation. Inspired by DIADEM, the NIH now hopes to receive authority to set up competitions of its own, says Landis, although she added that it was premature to say what kinds of problem the NIH might select as challenges.

When the results from the DIADEM competition are published in an upcoming special issue of Neuroinformatics, other researchers may in turn be inspired to join the mapping effort, says Ascoli. Given that the competition's data owners and algorithm developers are already brainstorming how to integrate their results into one research effort, he is hopeful that the technical barrier between them will soon fall.

"Once the first brick comes down," he says, "the whole wall follows."

Adam Mann 